\title{
Preparation of Poly(p-oxybenzoyl) Whisker in Paraffin Wax
}

\author{
Ashim Kumer SARKER, Kunio KImURA, ${ }^{\dagger}$ and Yuhiko YAMASHITA \\ Faculty of Environmental Science and Technology, Okayama University, \\ 3-1-1 Tsushima-naka, Okayama 700-8530, Japan
}

(Received December 7, 2001; Accepted April 22, 2002)

\begin{abstract}
An influence of solvent on morphology as well as growth mechanism of poly ( $p$-oxybenzoyl) whisker in paraffin wax (PW) was studied comparing with those in liquid paraffin (LPF). Furthermore, the condition for consecutive addition of oligomer was also examined to lengthen the whiskers in PW. Polymerizations of $p$-acetoxybenzoic acid were carried out in $\mathrm{PW}$ and $\mathrm{LPF}$ at $330^{\circ} \mathrm{C}$. Larger number of whiskers having smaller length and width were formed in PW compared with that in LPF. PW was a poorer solvent than LPF and this lower miscibility leaded to the crystallization of lower $\mathrm{DP}_{\mathrm{n}}$ oligomers. Additionally, formation rate of oligomer was bigger in PW than LPF. These facts resulted in the higher degree of super saturation of oligomers which generated a larger number of nuclei having smaller size. Since the length was determined by the number of nuclei and the yield, the length eventually became shorter. On the basis of the formation mechanism of the whisker in PW, oligomers were added into polymerization system to lengthen the whisker. When oligomers were added to compensate the consumed oligomers while the tip angle was constantly kept at $80^{\circ} \mathrm{C}$, the steady growth period was expanded and the length increased from $13.4 \mu \mathrm{m}$ to $22.1 \mu \mathrm{m}$.

KEY WORDS Poly(p-oxybenzoyl) / Whisker / Paraffin Wax / Crystallization / Oligomer / Miscibility /
\end{abstract}

Aromatic polyesters are characterized by the unique combination of thermal and mechanical properties and by a remarkable chemical resistance, which makes them useful high performance materials for various industrial applications such as reinforcement of composites, substitutions for asbestos and so on. However, wholly aromatic polyesters consisted of rigid-rod molecules show usually neither solubility or metlability under their decomposition temperatures. This intractability brings them about severe problems in industrial usage. Many attempts have been conducted with the aim of improving the poor processability from the point of both chemical modifications ${ }^{1}$ and processing techniques. $^{2}$

$\operatorname{Poly}(p$-oxybenzoyl) (POB) is a wholly aromatic polyester consisted of rigid-rod molecule and it is a hopeful candidate as high performance materials. POB has the similar problem derived from its intractability, and hence many thermotropic polyesters composed of $p$-oxybenzoyl unit as well as other aromatic copolyesters have been developed thus far. ${ }^{3-9}$ Thermotropic polyesters composed of $p$-oxybenzoyl unit are modified to reduce the chain rigidity by copolymerization, and therefore sacrifices the eventual properties predicted from the chain structure of POB. We have succeeded in obtaining whiskers of $\mathrm{POB}$ and other aromatic polyesters using crystallization of oligomers during solution polymerization. ${ }^{10-19} \mathrm{POB}$ whiskers are prepared from $p$-acetoxybenzoic acid ( $p$-ABA) in poor solvent such as liquid paraffin (LPF) with the elimina- tion of acetic acid at high temperature solution polymerization, of which the average length is $c a .40 \mu \mathrm{m}$ and the average width is $1.5 \mu \mathrm{m}$. These whiskers exhibit extended-chain single crystal in nature and polymer chains are oriented along the long axis of the whiskers. From the morphological observation of POB whiskers, it has been proposed that the formation mechanism of these whiskers bears the following steps; (1) The resulting oligomers are crystallized in the form of lamellae from solution polymerization as well as lamellae stack up to the long axis of the whisker with spiral growth caused by screw dislocation. (2) The eventual whiskers are accomplished with the increase of degree of polymerization by post polymerization in the interlamellar regions and reorganization of crystals occurs. This preparation technique stipulated a very useful method for morphology control of intractable aromatic polyester and many other aromatic whiskers have been reported. ${ }^{20-26}$

The length control of the whiskers is of great importance in industrial application. We had developed the method to lengthen the POB whiskers by the addition of oligomers and the whiskers having the longer length than $40 \mu \mathrm{m}$ can be tailored. ${ }^{27}$ Recently, it is very desirable to develop the control method of the length from 10 to $30 \mu \mathrm{m}$. The shorter whiskers are more valuable to be used as reinforcements of composites for precision instruments. We had previously studied on the solvent effect on the shape of the POB whisker and succeeded in preparing the shorter whiskers than $40 \mu \mathrm{m}$ in paraffin

${ }^{\dagger}$ To whom correspondence should be addressed (Tel \& Fax: +81-86-251-8902, E-mail: polykim@cc.okayama-u.ac.jp). 
wax (PW). ${ }^{16}$ The length of the whiskers were tunable from 10 to $40 \mu \mathrm{m}$ by the mixing ratio of PW and LPF. However, the width of the whisker also increased with the length because the miscibility between the solvent and the oligomer determined unequivocally the size of the whisker. ${ }^{28}$ This tendency is unfavorable for the aspect ratio of the whisker affecting the performance of the reinforcement and the control method by using the solvent is not suitable to lengthen the whisker without the increase of the width.

In this study, we clarify the details of the growth feature of the POB whisker in PW comparing with those in LPF and then examine the condition for consecutive addition of oligomer to tune its length in the range from 10 to $30 \mu \mathrm{m}$ with keeping its width.

\section{EXPERIMENTAL}

\section{Materials}

$p$-ABA was purchased from Tokyo Kasei Co. Ltd. and purified by recrystallization from ethyl acetate. LPF was purchased from Nakalai Tesque Co. Ltd and purified by vacuum distillation $\left(220^{\circ} \mathrm{C}-\right.$ $240^{\circ} \mathrm{C} / 0.2 \mathrm{mmHg}$ ). Paraffin wax $155\left(\mathrm{PW}, T_{\mathrm{m}}=69^{\circ} \mathrm{C}\right.$ ) was a gift from Nippon Seirou Co. Ltd. and used as received.

\section{Polymerization Method}

Preparation of Whisker. p-ABA $(0.9 \mathrm{~g})$ and PW $(47.0 \mathrm{~g}, 60 \mathrm{~mL}$ when it was melted) were placed into a cylindrical polymerization reactor equipped with a thermometer, a mechanical stirrer, and gas inlet and outlet tubes. This reaction mixture was heated. The mixture was stirred when PW was melted. When $p$-ABA was completely dissolved, the stirring was stopped. Then the mixture was heated up to $330^{\circ} \mathrm{C}$ and maintained at this temperature under a slow stream of nitrogen. In a couple of minutes, this reaction solution became turbid. The reaction was continued at $330^{\circ} \mathrm{C}$ for $6 \mathrm{~h}$ under a slow stream of nitrogen. The whiskers were collected by filtration at $330^{\circ} \mathrm{C}$, washed several times with hot toluene and then dried in a vacuum oven at $50^{\circ} \mathrm{C}$ for overnight. The filtrate was allowed to cool to room temperature and precipitated oligomers were collected by removal of $\mathrm{PW}$ with hot cyclohexane and then dried in a vacuum at $50^{\circ} \mathrm{C}$ for overnight.

Addition of Oligomer. In another polymerization reactor, $p$-ABA $(1.5 \mathrm{~g})$ and $\mathrm{PW}(7.8 \mathrm{~g}, 10 \mathrm{~mL}$ when it was melted) were placed and heating was continued up to $250^{\circ} \mathrm{C}$ under a slow stream of nitrogen. Then the stirring was done for a very few time after the monomer was completely dissolved. This solution $(1.6 \mathrm{~mL})$ was added into polymerization system at $10 \mathrm{~min}$ and $20 \mathrm{~min}$ after the reaction solution became turbid. The reaction was continued at $330^{\circ} \mathrm{C}$ for $6 \mathrm{~h}$ under a slow stream of nitrogen. The eventual whiskers were collected by the same procedures as described above.

\section{Synthesis of Oligomer Model Compound}

Phenyl[4-(4-benzoyloxy)benzoyloxy]benzoate. $p$ Hydroxybenzoic acid $(10.0 \mathrm{~g}, 0.0724 \mathrm{~mol})$, triethylamine $(16.12 \mathrm{~g}, 0.159 \mathrm{~mol})$ and $60 \mathrm{~mL}$ of distilled tetrahydrofuran were taken into a flask equipped with a thermometer, a dropping funnel and gas inlet and outlet tubes under a slow stream of nitrogen. Benzoyl chloride $(10.17 \mathrm{~g}, 0.0724 \mathrm{~mol})$ in $10 \mathrm{~mL}$ of distilled tetrahydrofuran was added dropwise from a dropping funnel into the reaction mixture at $0-5^{\circ} \mathrm{C}$ for $30 \mathrm{~min}$. The reaction was performed at $5^{\circ} \mathrm{C}$ for $2 \mathrm{~h}$ with continuous stirring and then $12 \mathrm{~h}$ at room temperature. Hydrochlorde-triethylamine salts were filtrated and tetrahydrofuran was evaporated. The product was washed with dilute hydrochloric acid and water. 4-Benzoyloxybenzoic acid (1.8 g) was obtained after recrystallization from the mixture of ethyl acetate and $n$-hexane. Then 4-benzoyloxybenzoyl chloride was synthesized from 4-benzoyloxybenzoic acid with thionyl chloride and reacted with 4-hydroxybenzoic acid phenyl ester by the similar procedure described above. Phenyl[4-(4-benzoyloxy)benzoyloxy]benzoate $(1.24 \mathrm{~g})$ was obtained after recrystallization from ethyl acetate (yield $60.5 \%$ ). Melting point was $173^{\circ} \mathrm{C}$. Elemental Analysis: Calcd for $\mathrm{C}_{27} \mathrm{H}_{18} \mathrm{O}_{6}$ : C, $73.97 \%$; $\mathrm{H}, 4.14 \%$. Found: C, $73.93 \%$; H, $4.12 \%$, IR $(\mathrm{KBr}$; $\left.\mathrm{cm}^{-1}\right): 2960,2925,2850,1743,1598,1509,1274$, $1207,1162,1061,1018,887,802,758,700,{ }^{1} \mathrm{H}$ NMR $\left(500 \mathrm{MHz}, \mathrm{CDCl}_{3} / \mathrm{CF}_{3} \mathrm{COOH}\right) \delta=7.73(\mathrm{H}-1, \mathrm{tr}, 1 \mathrm{H})$, $7.58(\mathrm{H}-2, \mathrm{tr}, 2 \mathrm{H}), 8.25(\mathrm{H}-3, \mathrm{~d}, 2 \mathrm{H}), 7.42(\mathrm{H}-6, \mathrm{~d}, 2 \mathrm{H})$ or $7.44(\mathrm{H}-6, \mathrm{~d}, 2 \mathrm{H}), 8.35(\mathrm{H}-7, \mathrm{~d}, 2 \mathrm{H})$ or $8.34(\mathrm{H}-$ $7, \mathrm{~d}, 2 \mathrm{H}), 7.44(\mathrm{H}-10, \mathrm{~d}, 2 \mathrm{H})$ or $7.42(\mathrm{H}-10, \mathrm{~d}, 2 \mathrm{H})$, $8.34(\mathrm{H}-11, \mathrm{~d}, 2 \mathrm{H})$ or $8.35(\mathrm{H}-11, \mathrm{~d}, 2 \mathrm{H}), 7.22(\mathrm{H}-14$, d, $2 \mathrm{H}), 7.47(\mathrm{H}-15, \mathrm{tr}, 2 \mathrm{H}), 7.34 \mathrm{ppm}(\mathrm{H}-16, \mathrm{tr}, 1 \mathrm{H})$, ${ }^{13} \mathrm{C} \mathrm{NMR}\left(125 \mathrm{MHz}, \mathrm{CDCl}_{3} / \mathrm{CF}_{3} \mathrm{COOH}\right) \delta=134.8(\mathrm{C}-$ 1), $129.0(\mathrm{C}-2), 130.5$ (C-3), 128.2 (C-4), 155.5 or 155.3 (C-5), 122.4 or 122.3 (C-6), 132.4 (C-7), 126.9 or $126.4(\mathrm{C}-8), 155.3$ or $155.5(\mathrm{C}-9), 122.3$ or 122.4 (C-10), $132.4(\mathrm{C}-11), 126.4$ or 126.9 (C-12), 150.4 (C13), 121.5 (C-14), 129.8 (C-15), 126.7 (C-16), 165.4 (C-17), 166.8 or 166.6 (C-18), 166.6 or 166.8 ppm (C19).

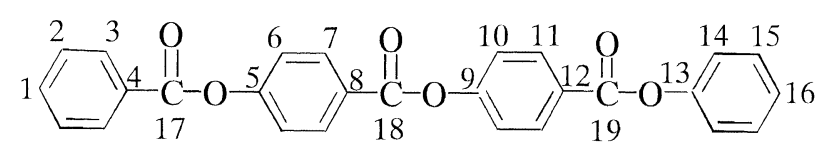




\section{Measurements}

Morphological observation was performed by scanning electron microscopy (SEM) using Hitachi S-2150. ${ }^{1} \mathrm{H}$ and ${ }^{13} \mathrm{C}$ NMR spectra were measured on Bruker AVANCE 500.

Oligomers dissolved in solution were analyzed by HPLC using Waters 490E HPLC system with Nova Pack HR C18 column. The mixture of acetic acid and acetnitril was used as an eluent and the mixing ratio of two solvent was changed from $90 / 10$ to $0 / 100$ by volume within $40 \mathrm{~min}$.

Oligomer concentration in polymerization solution was determined as follows. Remaining oligomers in filtrate were collected and hydrolyzed by $7.0 \% \mathrm{KOH}$ methanol solution. Then the solution was neutralized with dilute hydrochloric acid. $p$-Hydroxybenzoic acid and acetic acid were analyzed by gas chromatography (GC-14B Shimadzu) with FID equipped with a Thermon-300 in glass column. The number average degree of polymerization $\left(\mathrm{DP}_{\mathrm{n}}\right)$ of oligomers was calculated and then concentration of oligomers evaluated with $p$-oxybenzoyl unit was estimated by using the following eq 1 :

Concentration of oligomer in solution based on

$$
\begin{aligned}
& p \text {-oxybenzoyl unit }\left(\mathrm{mol} \mathrm{L}^{-1}\right) \\
& =\left\{\text { oligomer weight } /\left(\mathrm{DP}_{\mathrm{n}} \times 120+60\right)\right\} \\
& \times \mathrm{DP}_{\mathrm{n}} \times(1000 / 60)
\end{aligned}
$$

In order to compare the growth rates on the spiral and on the lateral side, the average shape parameters of the whiskers were estimated from morphological observations in the course of polymerization. From the parameters obtained, the volume of whiskers was calculated by using the following equation:

$$
\text { Volume of whiskers }\left(\mu \mathrm{m}^{3}\right)=(\sqrt{3} / 8) W^{2}(L+2 l)
$$

Where $W$ is the average width of the whisker, $L$ is the average length of the whiskers and $l$ is the average trunk length of the whiskers. $W$ and $L$ were determined by taking the average of over 60 observation values. The increased volumes with the spiral growth and with the lateral growth per growth surface area were calculated according to the illustrated drawing in Scheme. The quantity of crystallized oligomers was evaluated with p-oxybenzoyl unit.

\section{Preparation of Concentration-temperature (C-T) Phase Diagram of Oligomer Model Compound}

Oligomer model compound and solvent were put into glass tubes at different concentrations. They were placed into oil bath and heated until the oligomer model compound was completely dissolved. Then temperature was lowered gradually at a rate of $5^{\circ} \mathrm{C} \mathrm{h}^{-1}$ and

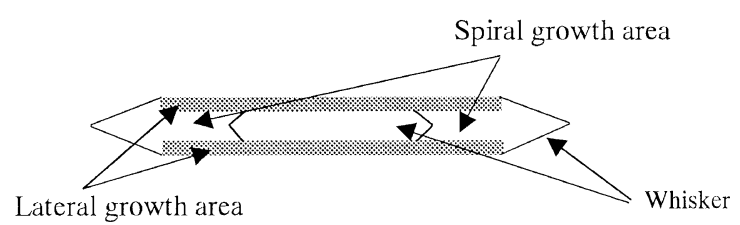

Scheme. Definition of the spiral growth and the lateral growth surface area used to calculate crystallized oligomers.

the cloud point temperature was determined. The phases and conjugate line between two immiscible liquid phase and liquid-solid phase were confirmed by microscope equipped with heating stage (MP-500D, Yanaco Co. Ltd.) under crossed polarization.

\section{RESULTS AND DISCUSSION}

\section{Morphological Characteristics of POB Whiskers}

Polymerizations were carried out at $330^{\circ} \mathrm{C}$ in $\mathrm{LPF}$ and PW. Polymerization concentration was $1.0 \%$ based on polymer weight. LPF is a branched paraffin which contains $15.2 \%$ of tertiary carbon analyzed by ${ }^{13} \mathrm{C}$ NMR and its molecular weight is 928 . PW is a completely linear solid paraffin, of which molecular weight is 472 . The polymerization results are summarized in Table I and the morphologies of the obtained crystals are shown in Figure 1. Whiskers are obtained in both solvents. The length and width of whisker prepared in LPF (POB-1) are 40.3 and $1.1 \mu \mathrm{m}$, respectively. On the other hand, those of whisker prepared in PW (POB-2) are 13.4 and $0.9 \mu \mathrm{m}$, respectively. The whisker prepared in PW is much smaller than that in LPF. The both tips of POB-1 are very sharp but those of POB-2 are almost flat. POB-2 shows radial growth nature from the center of the whisker. The number of crystals calculated from the yield, density and the size parameter as reported previously ${ }^{14}$ is 3 times larger in PW than LPF. The yields in both polymerization system are not quantitative. The oligomers did not remain at all after $6 \mathrm{~h}$ polymerization and this low yield is due to the sublimation of $p$-ABA during polymerization, especially in the initial stage of polymerization. $p$-ABA sublimates easier in PW. It is found that more whiskers having shorter length and width are formed in PW, which must be attributed to the formation of much more nuclei having smaller width compared with those in LPF.

It is well known that critical radius of nucleus $\left(r^{*}\right)$ and nucleation rate $(J)$ depend on the degree of super saturation as follows. ${ }^{29,30}$

$$
\begin{aligned}
& \Delta \mu=k T \ln (1+\sigma) \quad \sigma=(C-C e) / C e \\
& r^{*}=2 v \gamma / \Delta \mu \\
& J=v_{+} q \exp \left(-\Delta G^{*} / k T\right) \\
& \quad=v_{+} q \exp \left(-16 \pi \gamma^{3} v^{2} / 3 \Delta \mu^{2} k T\right)
\end{aligned}
$$


Table I. Results of polymerization ${ }^{\mathrm{a}}$

\begin{tabular}{|c|c|c|c|c|c|c|c|}
\hline \multirow{2}{*}{ Polymer } & \multirow{2}{*}{ Solvent } & \multirow{2}{*}{$\frac{T_{\mathrm{s}}^{\mathrm{b}}}{{ }^{\circ} \mathrm{C}}$} & \multirow{2}{*}{$\frac{t_{\mathrm{t}}^{\mathrm{c}}}{\min }$} & \multirow{2}{*}{$\frac{\text { Yield }}{\%}$} & \multicolumn{2}{|c|}{ Size of whiskers $(\mu \mathrm{m})$} & \multirow{2}{*}{$\begin{array}{c}\text { Number of whisker } \\
\text { in } 60 \mathrm{~mL}\end{array}$} \\
\hline & & & & & Length & Width & \\
\hline POB-1 & LPF & 215 & 2.0 & 43.3 & 40.3 & 1.1 & $0.78 \times 10^{10}$ \\
\hline POB-2 & PW & 226 & 1.5 & 39.2 & 13.4 & 0.9 & $2.43 \times 10^{10}$ \\
\hline
\end{tabular}

${ }^{\mathrm{a}}$ Polymerization was carried out at $330^{\circ} \mathrm{C}$ and a concentration of $1.5(\mathrm{wt} / \mathrm{vol}) \%$ for $6 \mathrm{~h} .{ }^{\mathrm{b}}$ Temperature at which the monomer was dissolved completely. ${ }^{\mathrm{c}}$ Time when the solution became turbid.

(a)

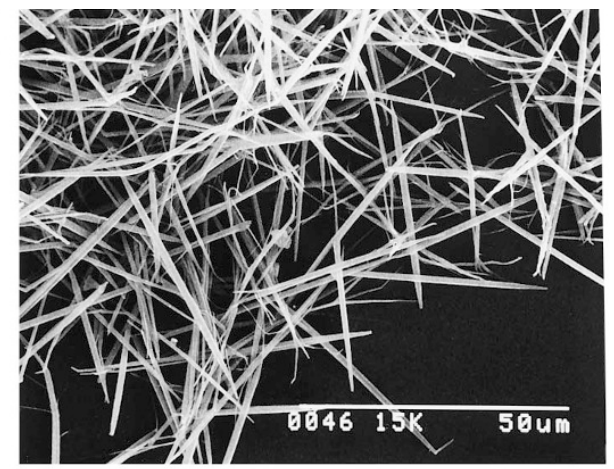

(b)

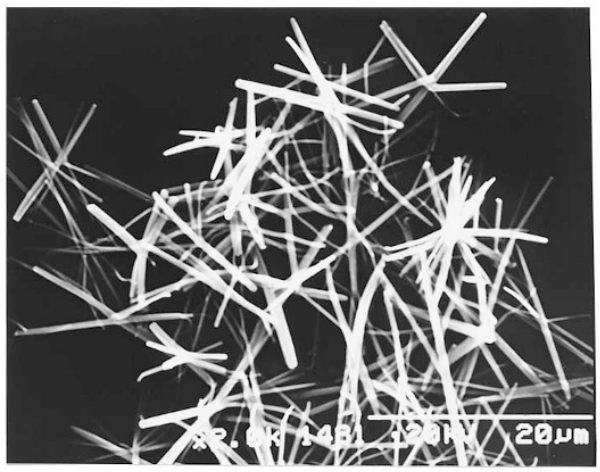

Figure 1. SEM of POB whiskers prepared in (a) LPF (POB-1) and (b) PW (POB-2).

where $\mu$ : chemical potential

$C$ : concentration of solute

$\mathrm{Ce}$ : equilibrium concentration

$r^{*}$ : critical radius of nucleus

$v$ : volume of molecule

$\gamma:$ density of surface energy

$J$ : nucleation rate

$v_{+}$: rate of crystallization of one molecule into critical nucleus

$q$ : density of free molecule

When the degree of super saturation increases, $\Delta \mu$ which is a driving force for nucleation becomes larger. This large $\Delta \mu$ leads to small $r^{*}$ and large $J$ which means that the much more nuclei having smaller radius are formed. There seems to exist several parameters to influence the degree of super saturation in the case of this study such as miscibility between oligomer and solvent, formation rate of oligomer, concentration, temperature and so on. Polymerizations were carried out at the same concentration and temperature, and thereby former two parameters are more dominant.
Firstly, miscibility between oligomer and solvent is discussed. The temperature at which $p$-ABA is completely dissolved $\left(T_{\mathrm{S}}\right)$ during heating and the time when the solution becomes turbid $\left(t_{\mathrm{t}}\right)$ are adaptable as criterion to estimate the miscibility between oligomer and solvent, and lower $T_{\mathrm{s}}$ and later $t_{\mathrm{t}}$ suggest higher miscibility. $T_{\mathrm{s}}$ in PW is higher and $t_{\mathrm{t}}$ is earlier than those in LPF. Hereby, the miscibility between the oligomer and $\mathrm{PW}$ is lower. Comparison of $C-T$ phase diagrams of oligomer and the solvents gives very useful information about the miscibility in order to confirm the above discussion. But the phase diagram of oligomer cannot be accurately determined because the phase-separated oligomers are insoluble into common organic solvents under the temperature at which the polymerization starts and the phase-separated oligomers cannot be isolated. In this study, the miscibility between oligomer and solvents is discussed by a clue of the miscibility of oligomer model compound and solvent. Phenyl[4-(4benzoyloxy)benzoyloxy]benzoate was synthesized as the oligomer model compound which was end-capped by phenyl group to avoid an influence of polymerization during preparation of phase diagrams. $C-T$ phase diagrams prepared by the cloud point measurements are shown in Figure 2. Reaction-induced phase separation of oligomers in poor solvent is describable on the analogous $C-T$ phase diagram to that of partially miscible polymer-solvent system. ${ }^{31,32}$ The phase separation curve in the repulsive system in which there is no attractive interaction between oligomer and solvent is written as the combination of the freezing point curve of oligomers and the UCST type consolution curve. In the diagrams of oligomer model compound and solvent, the phase separation appears as the combination of the freezing point curve of model compound and the UCST type consolution curve as described above. The phase separation curve in PW system shifts towards higher temperature than that in LPF system and it becomes clear that the miscibility between oligomer and PW is lower than that in LPF. This result confirms the above discussion.

Secondary, formation rate of oligomer is examined in both solvents. Polymerization kinetics in both solvents is followed by the titration of by-produced acetic acid with $\mathrm{NaOH}$ solution. The reaction of $p$-ABA obeys the 


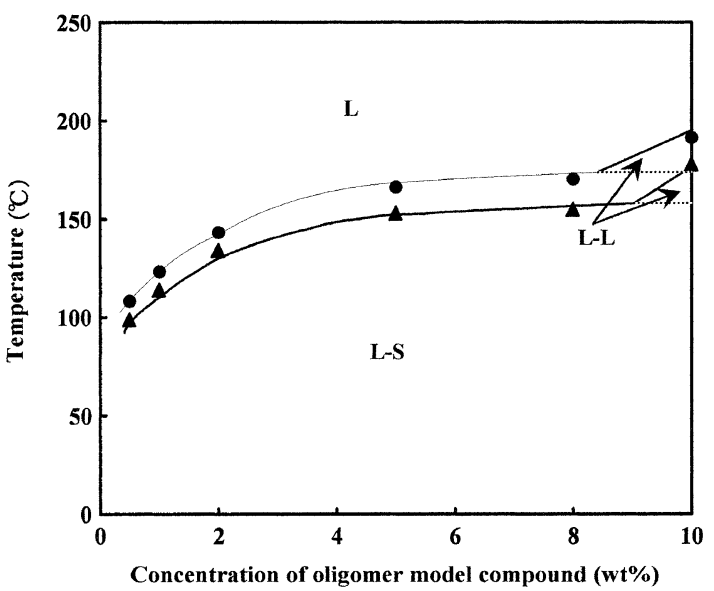

Figure 2. $C$ - $T$ phase diagram for the system of oligomer model

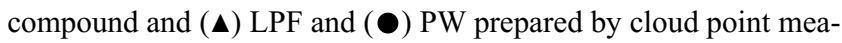
surement. L: Liquid region, L-L: Two liquid region, L-S: Liquidsolid region.

Table II. Polymerization rate constants and $\mathrm{DP}_{\mathrm{n}}$ of oligomers in LPF and PW

\begin{tabular}{lccc}
\hline Solvent & $\begin{array}{c}\text { Rate constant } \\
\text { of } k 2\left(\mathrm{~L} \mathrm{~mol}^{-1} \mathrm{~min}^{-1}\right)\end{array}$ & avg. $\mathrm{DP}_{\mathrm{n}}{ }^{\mathrm{a}}$ & ps. $\mathrm{DP}_{\mathrm{n}}{ }^{\mathrm{b}}$ \\
\hline LPF & 0.55 & 1.16 & 8 \\
PW & 0.71 & 1.09 & 7 \\
\hline
\end{tabular}

${ }^{\mathrm{a}}$ Average $\mathrm{DP}_{\mathrm{n}}$ of oligomer when the solution became turbid was determined by titration of by- produced acetic acid. ${ }^{b} \mathrm{DP}_{\mathrm{n}}$ of phase-separated oligomer estimated by HPLC.

secondary order kinetics in both solvents prior to the beginning of crystallization. The rate constants and average $\mathrm{DP}_{\mathrm{n}}$ of oligomer when the solution became turbid are presented in Table II. The polymerization rate is about 1.3 times higher in PW than in LPF. Average $\mathrm{DP}_{\mathrm{n}}$ of oligomers when the crystallization begins is lower in PW than that in LPF. This fact suggests that the lower oligomers are precipitated to crystallize in PW owing to lower miscibility. The oligomers dissolved in the solution were collected and analyzed by HPLC. The collected oligomers contain from $p$-ABA to hexamer and thereby the oligomers more than heptamer are crystallized in PW, which is smaller than that of octamer in LPF. ${ }^{12}$ It can be thought on the basis of these results that the degree of super saturation of oligomer in PW is significantly higher than that in LPF and a larger number of nuclei with smaller width are formed in PW. Hence, the length of the whisker becomes reasonably shorter in PW under the same yield as in LPF.

\section{Growth Feature POB Whiskers in PW}

The changes in size and shape of the whiskers prepared in PW are followed with polymerization to know the growth feature of the whiskers. Figure 3 shows the incipient crystals prepared in PW. The crystals prepared for 10 min exhibit the very similar shape to the incipi- (a)

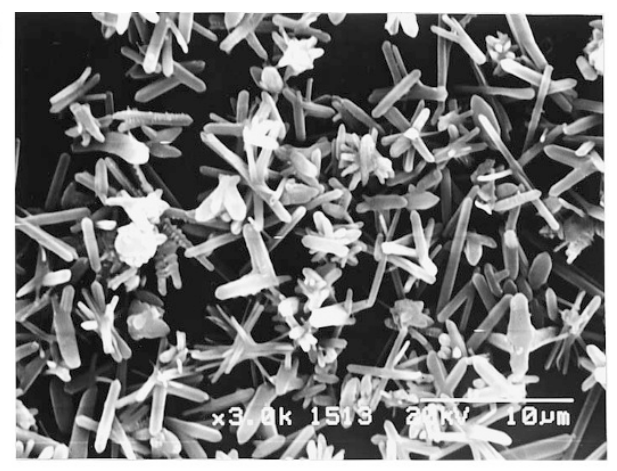

(b)

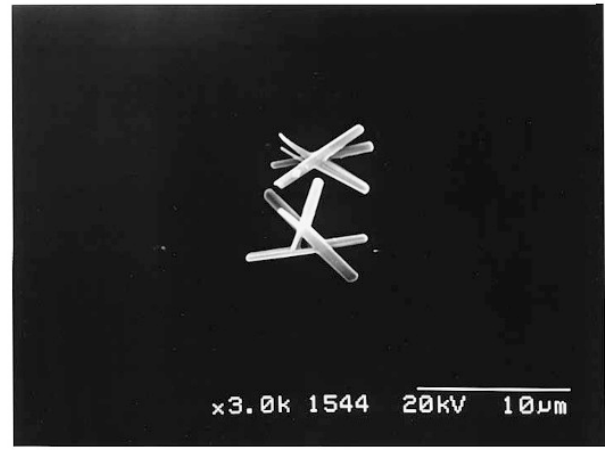

Figure 3. Incipient POB crystals prepared in PW obtained for (a) $10 \mathrm{~min}$ and (b) $30 \mathrm{~min}$.

ent crystals prepared in $\mathrm{LPF},{ }^{31}$ of which the cross section is hexagonal and tip angle is $80^{\circ}$. Only the size is very different, that is, the crystals prepared in PW are much smaller. The crystals show radial growth nature even at very early stage of polymerization, that is, several crystals are separately grown from the center part of the crystal. During the very rapid nucleation process many nuclei are formed at once and they might aggregate to generate interpenetrated or overgrown nuclei which lead to radial growth nature. It is found that the tip angle becomes flat in PW. The changes in the shape and size in the course of whisker growth are plotted in Figure 4. The length and width increase gradually with the yield and they are leveled off at $3 \mathrm{~h}$. This is an evidence that the whisker is growing by the consecutive supply of oligomers from the solution. The tip angle of whiskers is constant at $80^{\circ}$ up to $10 \mathrm{~min}$. Afterwards, it increases gradually and finally becomes flat. The trunk ratio of the whisker as defined in Figure 4 is calculated. The trunk ratio increases gradually with whisker growth, which is proportional to the tip angle. This is the opposite phenomenon in LPF, that is, sudden decrease of the trunk ratio at $15 \mathrm{~min}$ is caused by the sharpening of tip angle in LPF. ${ }^{31}$ The number of the whisker is almost constant during polymerization. Once the nuclei are generated in the solution, oligomers are predominantly crystallized onto the nuclei due to the advantage of surface energy and new nuclei are not generated in the course of the whisker growth. Further 

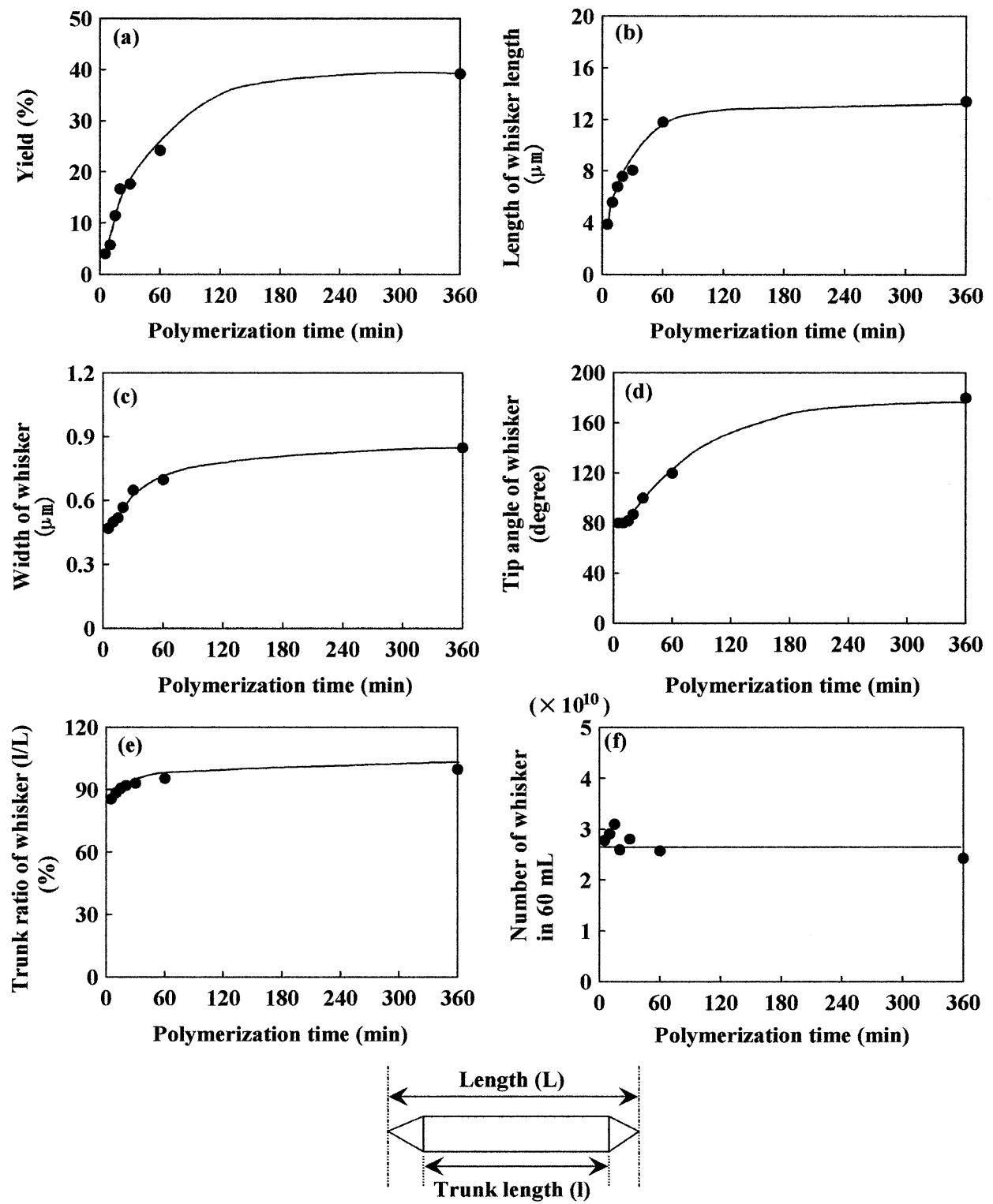

Figure 4. Polymerization time dependencies of (a) yield, (b) length, (c) width, (d) tip angle, (e) trunk ratio, and (f) number of POB whiskers prepared in PW.

study is required to elucidate the nature of the change in tip angle.

\section{Preparation of Longer Whiskers by Addition of Oligomer}

Addition of Consumed Oligomers During the Steady State Growth. As known, the spiral growth is very important for whisker preparation and the increase of whisker length is caused by which oligomer lamellae pile up along the long axis of whiskers with the spiral growth. In order to increase the length of the whiskers with keeping the width, consumed oligomers have to be supplied from outside into polymerization system to keep the critical degree of super saturation of oligomer and to expand the steady growth period as previously reported. ${ }^{31}$ The steady state of the whisker growth is achieved in a desirable balance between crystallization rate and formation rate of oligomer. The change in the tip angle is the most susceptible to that balance. If the balance is broken, tip angle cannot be constantly kept at $80^{\circ}$. In the case of polymerization in PW, tip angle becomes flat after $15 \mathrm{~min}$ and hereby the steady growth period is supposed to be up to $15 \mathrm{~min}$ from the beginning of crystallization. The oligomers were added twice into polymerization system at $10 \mathrm{~min}$ just before the tip angle became flat and then $20 \mathrm{~min}$ to compensate the decreased concentration of oligomers. The amount of consumed oligomer after 10 min calculated according to the eq 1 was $1.32 \mathrm{mmol}$ based on $p$-oxybenzoyl unit. The oligomer solution for the addition was prepared according to our previous paper. ${ }^{31}$ The results are summarized in Table III. The length of whiskers increased from 13.4 to $18.3 \mu \mathrm{m}$ by the first addition and to $22.1 \mu \mathrm{m}$ by the second addition. The changes in the 
Table III. Morphological features of POB whiskers obtained by addition of oligomer ${ }^{\mathrm{a}}$

\begin{tabular}{|c|c|c|c|c|c|}
\hline \multirow{2}{*}{ No. of addition } & \multirow{2}{*}{$\frac{\text { Yield }}{\%}$} & \multicolumn{2}{|c|}{ Size of whiskers $(\mu \mathrm{m})$} & \multirow{2}{*}{$\frac{\text { Tip angle }}{\text { degree }}$} & \multirow{2}{*}{$\begin{array}{l}\text { Number of whisker } \\
\text { in } 60 \mathrm{~mL}\end{array}$} \\
\hline & & Length & Width & & \\
\hline 0 & 39.2 & 13.4 & 0.85 & 180 & $2.4 \times 10^{10}$ \\
\hline $1^{\mathrm{b}}$ & 43.5 & 18.3 & 0.94 & 180 & $2.2 \times 10^{10}$ \\
\hline $2^{c}$ & 43.8 & 22.1 & 1.02 & 180 & $2.1 \times 10^{10}$ \\
\hline
\end{tabular}

${ }^{a}$ Polymerization was carried out in $\mathrm{PW}$ at $330^{\circ} \mathrm{C}$ for $6 \mathrm{~h} .{ }^{\mathrm{b}} 1.60 \mathrm{~mL}$ of $0.83 \mathrm{~mol} \mathrm{~L}{ }^{-1}$ oligomer solution was added into polymerization system at 10 min after the crystallization occurred. ${ }^{c} 1.60 \mathrm{~mL}$ of $0.83 \mathrm{~mol} \mathrm{~L}^{-1}$ oligomer solution was added into polymerization system at $10 \mathrm{~min}$ and $20 \mathrm{~min}$ after the crystallization occurred.
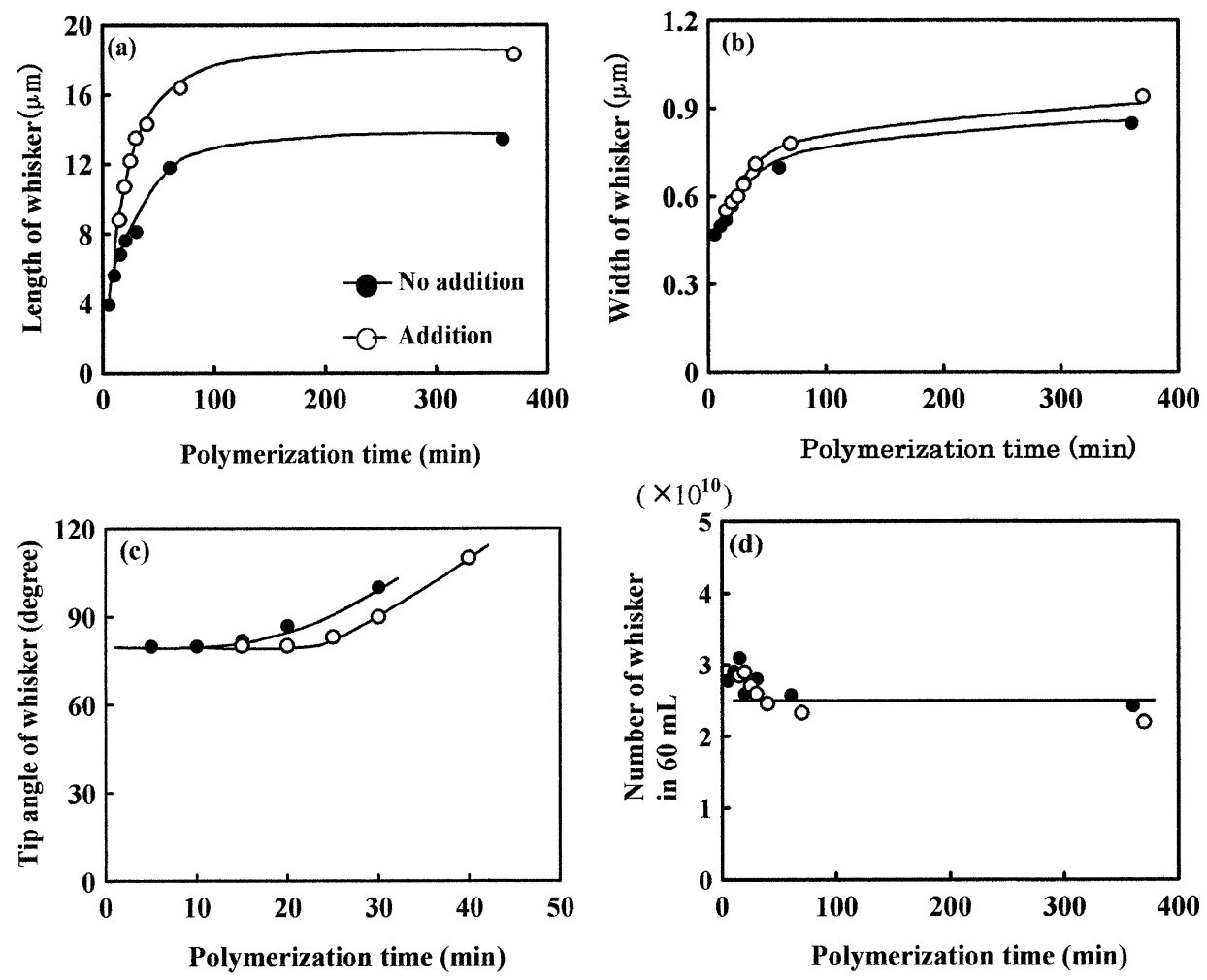

Figure 5. Polymerization time dependencies of (a) length, (b) width, (c) tip angle, and (d) number of POB whiskers prepared by the addition of oligomers at $10 \mathrm{~min}$.

length, tip angle, number and width of the whiskers by the first addition with polymerization time are shown in Figure 5. It is proved that the whiskers is lengthened by the addition of oligomers and the starting time of flattening of the tip becomes delayed as well as the steady state growth period is expanded. The number of whiskers remains almost constant after the addition of oligomers and nucleation is not caused by the addition of oligomers. From these results, it can be concluded that if the degree of super saturation of oligomer is constantly kept, the length of whisker increases. The width increases slightly from 0.85 to $1.02 \mu \mathrm{m}$ by the addition of oligomers in this experiment. This result shows that the whiskers grow not only with spiral growth but also with a slight lateral side growth. The reason for the slight lateral growth is due to the high degree of super saturation of oligomers which increases temporarily just after the addition of oligomers. The lateral growth of whisker must be prevented by adding the oligomers a little at a time. The quantities of crystallized oligomers per growth surface area are examined in the spiral and the lateral side with polymerization time. Table IV ascertained that the quantity of crystallized oligomers in the spiral growth per growth surface area is much larger than that in the lateral side growth. Oligomers crystallize more preferentially on the spiral step rather than the lateral side.

\section{Addition of Consumed Oligomers after Flattening of} Tip. Oligomers were added at $30 \mathrm{~min}$ after the crystallization began, when the tip angle was $90^{\circ} .30 \mathrm{~min}$ is out of the steady growth period. Figure 6 shows the morphology of the whiskers prepared for $15 \mathrm{~min}$ after the addition of oligomers at $30 \mathrm{~min}$. There observes many small whiskers which are recognized as newly formed whiskers. The change in the length, width and tip angle of the whiskers with polymerization time are shown in Figure 7. Two kinds of whiskers having different size exist clearly, which are corresponding to al- 
Table IV. Quantity of crystallized oligomers in spiral and lateral side in polymerization system of addition of oligomer

\begin{tabular}{|c|c|c|c|c|}
\hline $\begin{array}{l}\text { Addition } \\
\frac{\text { time }}{\min }\end{array}$ & $\frac{\text { Time }^{\mathrm{a}}}{\mathrm{min}}$ & $\begin{array}{l}\text { Crystallized oligomers in } \\
\text { spiral growth per growth } \\
\frac{\text { surface area (Conc. } 1)^{\mathrm{b}}}{\mathrm{mol} \mu \mathrm{m}^{-2}}\end{array}$ & $\begin{array}{l}\text { Crystallized oligomers in } \\
\text { lateral growth per growth } \\
\frac{\text { surface area (Conc. } 2)^{\mathrm{b}}}{\mathrm{mol} \mathrm{um}^{-2}}\end{array}$ & $\begin{array}{c}\text { Ratio of } \\
\text { Conc. } 1 / \text { Conc. } 2\end{array}$ \\
\hline \multirow[t]{4}{*}{$10^{\mathrm{c}}$} & 5 & $11.6 \times 10^{-15}$ & $0.03 \times 10^{-15}$ & 386.7 \\
\hline & 10 & $20.3 \times 10^{-15}$ & $0.07 \times 10^{-15}$ & 290.0 \\
\hline & 15 & $27.9 \times 10^{-15}$ & $0.13 \times 10^{-15}$ & 214.6 \\
\hline & 30 & $49.4 \times 10^{-15}$ & $0.70 \times 10^{-15}$ & 70.0 \\
\hline \multirow[t]{4}{*}{$30^{\mathrm{d}}$} & 5 & $1.5 \times 10^{-15}$ & $0.01 \times 10^{-15}$ & 150.0 \\
\hline & 10 & $3.0 \times 10^{-15}$ & $0.06 \times 10^{-15}$ & 50.0 \\
\hline & 15 & $4.5 \times 10^{-15}$ & $0.15 \times 10^{-15}$ & 30.0 \\
\hline & 30 & $8.7 \times 10^{-15}$ & $0.50 \times 10^{-15}$ & 17.4 \\
\hline
\end{tabular}

\footnotetext{
${ }^{\mathrm{a}}$ Time after the addition of oligomer. ${ }^{\mathrm{b}}$ Described as concentration of $p$-oxybenzoyl unit. ${ }^{c} 1.6 \mathrm{~mL}$ of $0.83 \mathrm{~mol} \mathrm{\textrm {L } ^ { - 1 }}$ oligomer solution was added into polymerization system at 10 min after the crystallization occurred. ${ }^{d} 2.96 \mathrm{~mL}$ of 0.83 mol L ${ }^{-1}$ oligomer solution was added into polymerization system at $30 \mathrm{~min}$ after the crystallization occurred.
}

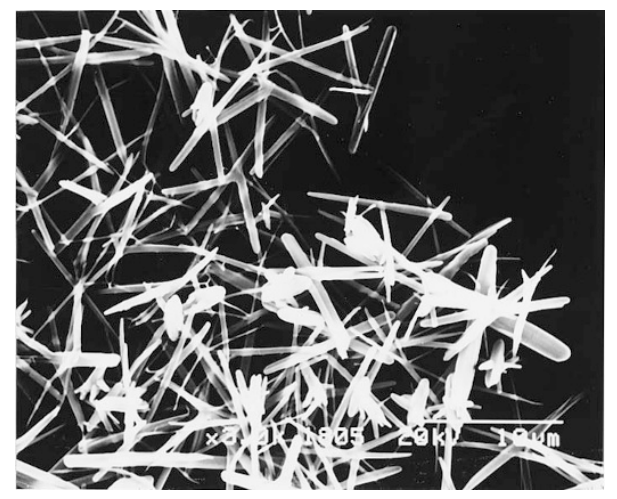

Figure 6. $\mathrm{POB}$ whiskers prepared in $\mathrm{PW}$ for $15 \mathrm{~min}$ after the addition of oligomers at $30 \mathrm{~min}$.

ready existed whiskers (old whiskers) and newly created whiskers (new whiskers), respectively. It becomes more clear by the change in the distribution diagrams of the length illustrated in Figure 8 compared with that prepared by the addition of oligomers at $10 \mathrm{~min}$. In the diagram of the whisker prepared by adding at $10 \mathrm{~min}$, the length increases and the distribution becomes broader as polymerization proceeds. On the other hand, a peak shifts toward shorter length at $5 \mathrm{~min}$ after the addition at $30 \mathrm{~min}$. This peak shift is a clear proof for the generation of new nuclei. The apparent rates of the increment of the length are estimated as shown in Figure 9. The increments of length of old whiskers and new whiskers become 10 times and 2 times smaller than that with the addition at $10 \mathrm{~min}$, respectively. On the other hand, the increments of width of old whiskers and new whiskers become 2 times and 4 times larger than that with the addition at $10 \mathrm{~min}$, respectively. Oligomers tend to crystallize more on the lateral side by the addition at $30 \mathrm{~min}$. Tip angle of old whiskers becomes almost flat. The percentage of newly created whiskers are about $75 \%$. Table IV ascertained that the quantity of crystallized oligomers in the spiral
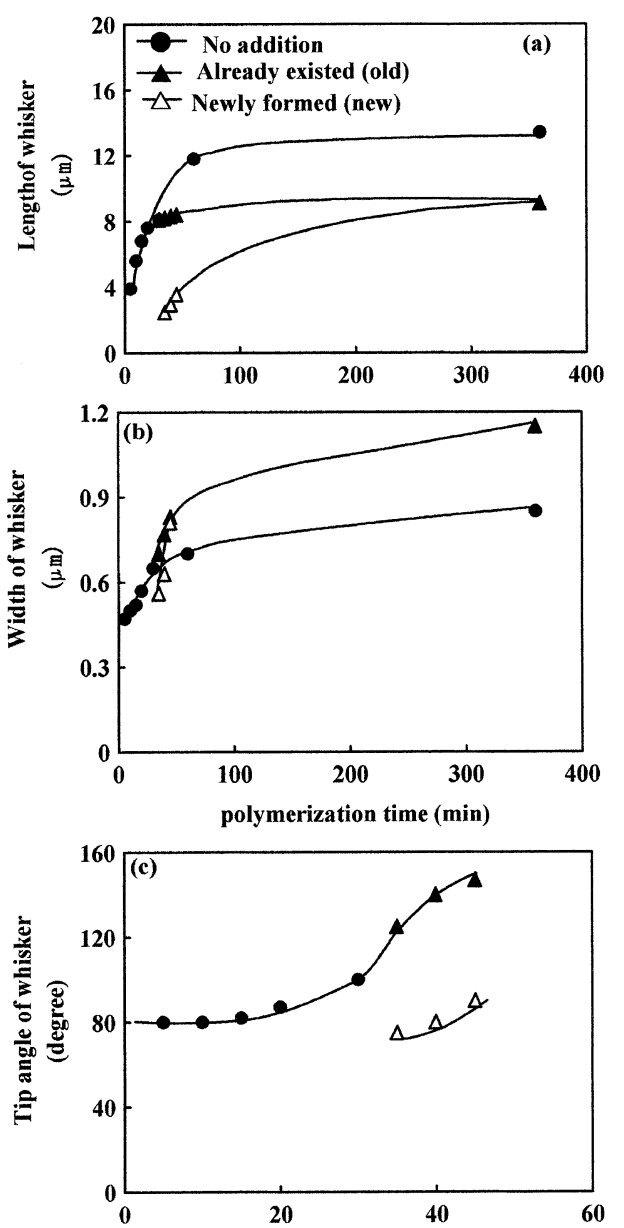

Figure 7. Polymerization time dependencies of (a) length, (b) width, and (c) tip angle of whiskers prepared in PW by addition of oligomers at $30 \mathrm{~min}$.

growth per growth surface area is also larger than that in the lateral side growth. Compared with the results by the addition at $10 \mathrm{~min}$, spiral growth is still more favorable than the lateral growth, but the quantity of crystallizable oligomers in both growths becomes closer. The length does not increase by the addition of oligomers 


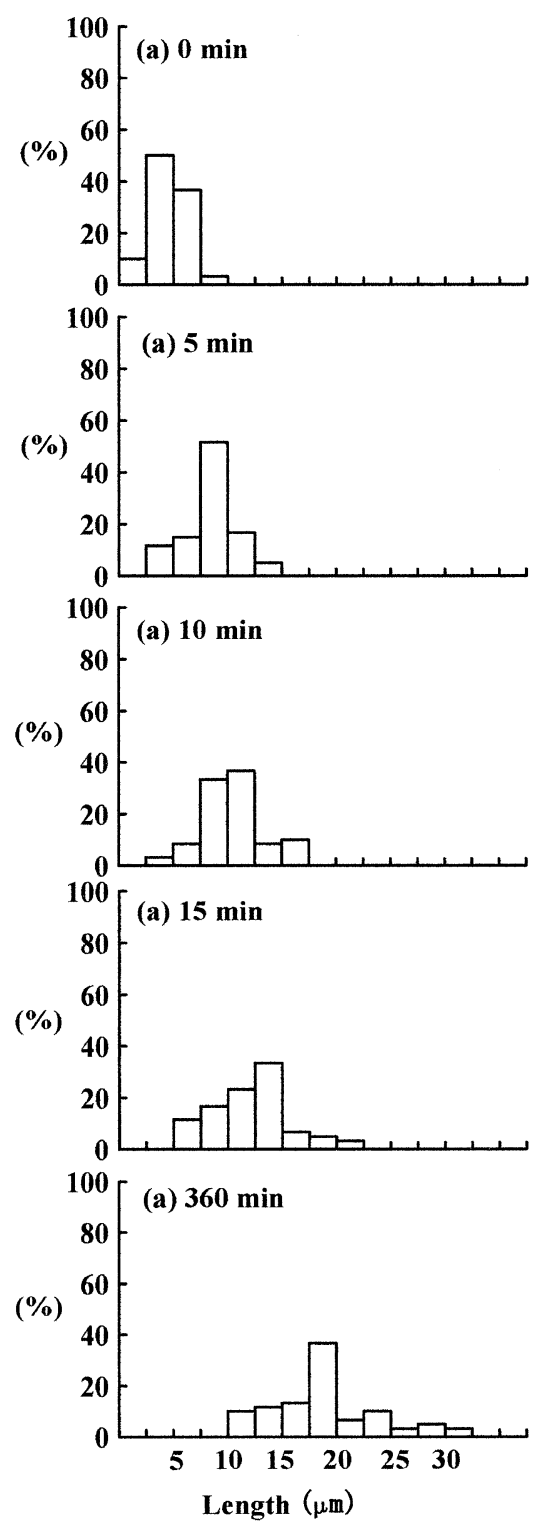

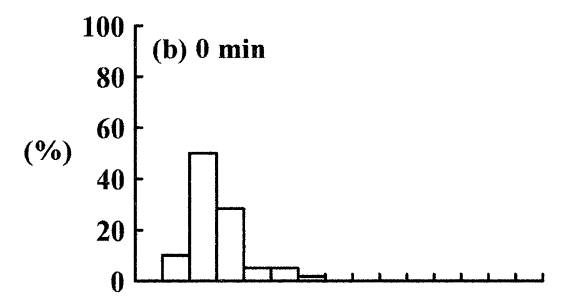
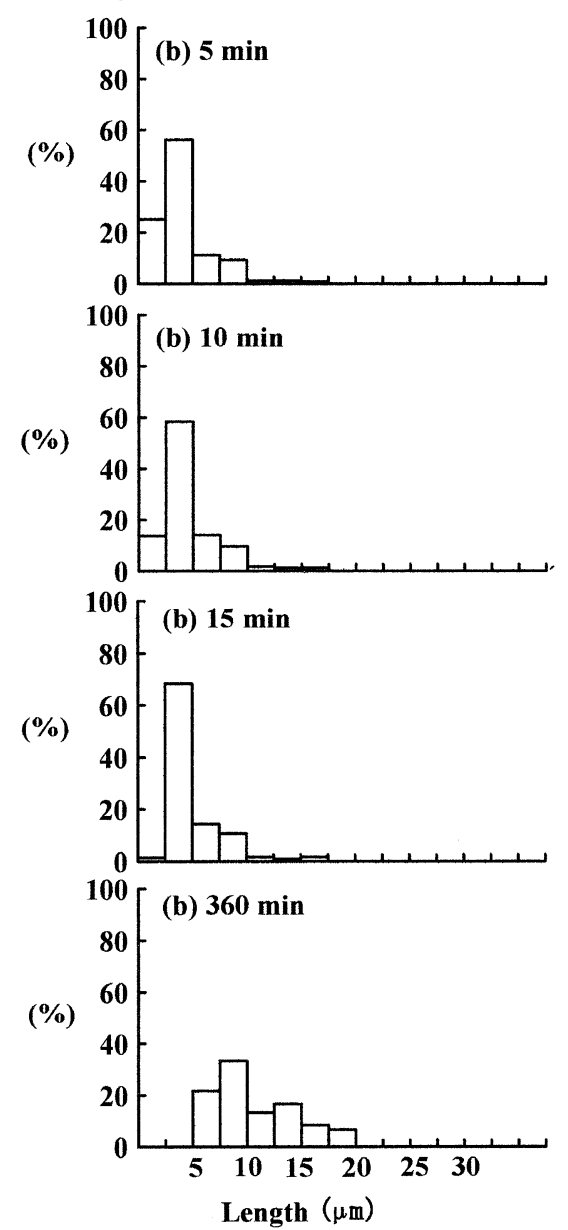

Figure 8. Distribution diagrams of length of POB whiskers prepared by the addition of oligomer at (a) $10 \mathrm{~min}$ and (b) $30 \mathrm{~min}$. Time in diagrams describes polymerization time after the addition of oligomers.

after steady growth period because the oligomers are consumed to generate new nuclei and lateral growth. In order to lengthen the whiskers, it can be concluded that consumed oligomers should be added into polymerization system when the tip angle is constantly kept at $80^{\circ}$.

\section{CONCLUSION}

In the polymerization of $p$-ABA in $\mathrm{PW}$, the lower $\mathrm{DP}_{\mathrm{n}}$ oligomers were crystallized due to the lower miscibility and the formation rate of oligomers was larger compared with that in LPF. Therefore, the degree of super saturation of oligomers in PW became higher and it led to the generation of a larger number of nuclei having smaller width. As a result, POB whiskers having smaller length and width were formed in PW. On the basis of the growth feature of the whisker in PW, oligomers were added into polymerization system from outside to lengthen the whisker. When the oligomers were added to compensate the consumed oligomers and keep the concentration of oligomer while the tip angle was constantly kept at $80^{\circ}$, the steady growth period was expanded and the length increased from 13.4 to $22.1 \mu \mathrm{m}$ with very slightly increase of the width from 0.85 to $1.02 \mu \mathrm{m}$. The length did not increase at all by the addition of oligomers after steady growth period because the oligomers were consumed to generate new nuclei and lateral growth. A combination of the polymerization in PW and the consecutive addition of oligomer is a favorable method to tune the length of the POB whisker in the range from 10 to $30 \mu \mathrm{m}$ with keeping its width. 

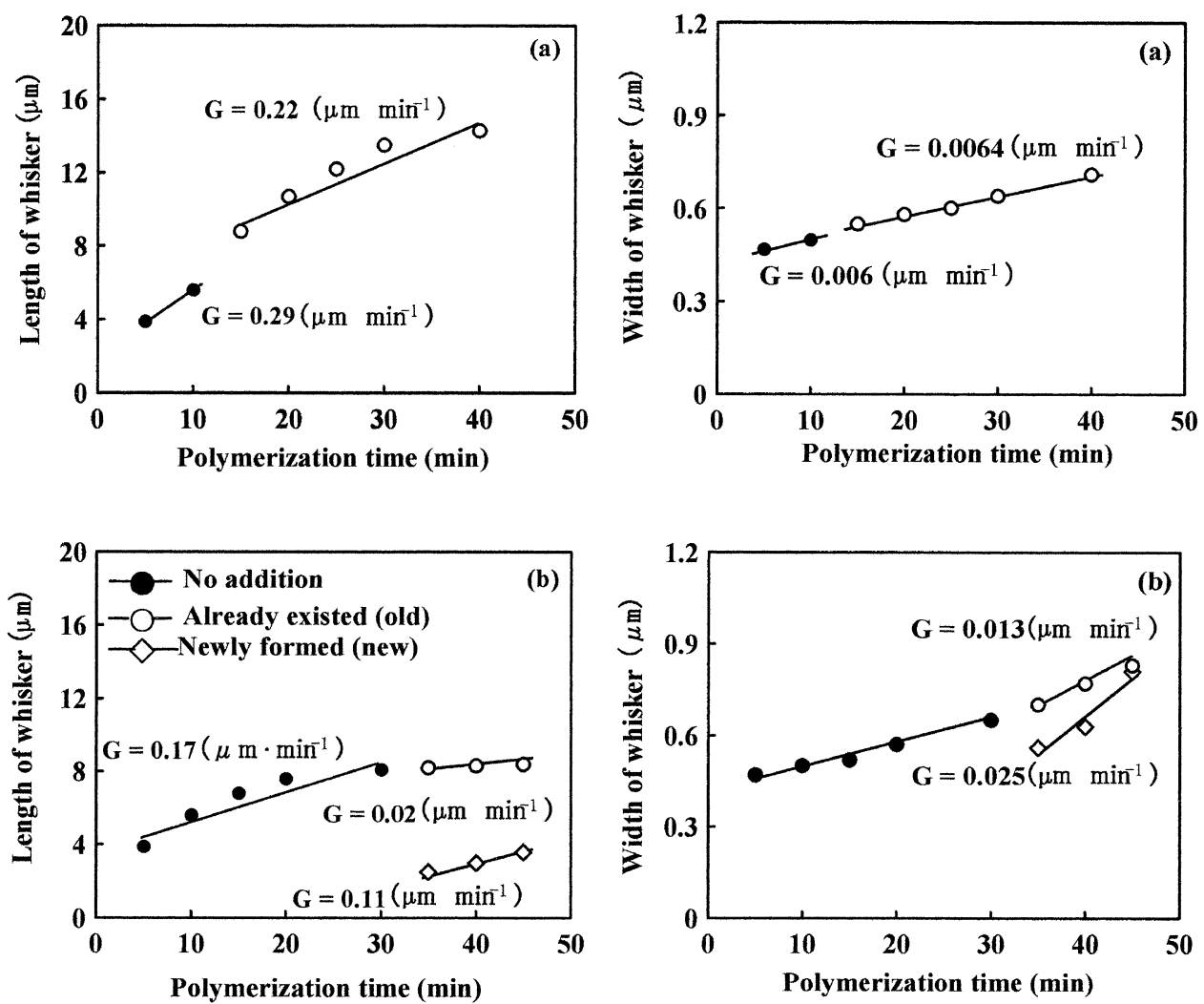

Figure 9. Initial increase of length of $\mathrm{POB}$ whiskers prepared by the addition of oligomer at (a) 10 min and (b) 30 min after the crystallization began.

\section{REFERENCES}

1. for example;

a) P. E. Cassidy, "Thermally Stable Polymers: Syntheses and Properties", Marcel Dekker, Inc., New York, N.Y., 1980.

b) A. G. Baird and A. Blumstein, "Liquid Crystalline Order in Polymers", London, Academic Press, Ltd., 1978.

2. for example;

R. B. Seymour and G. S. Kirshenbaum, "High Perform. Polym., Their Origin and Development", Proceedings of the Symposium on the History of High Performance Polymers, The American Chemical Society Meeting, New York, April 15-18, 1986, Elsevier, London, 1986.

3. R. Gilkey and J. R. Caldwell, J. Appl. Polym. Sci., 2, 198 (1959).

4. J. Economy, R. S. Storm, V. I. Matkovich, S. G. Cottis, and B. E. Nowak, J. Polym. Sci., Polym. Chem. Ed., 14, 2207 (1976).

5. J. Economy, W. Volksen, and R. H. Geiss, Mol. Cryst. Liq. Cryst., 105, 289 (1984).

6. A. Flores, F. A. Balta, F. J. Calleja, and H. R. Kricheldorf, J. Mater. Sci. Lett., 14, 1571 (1995).

7. W. J. Jackson, Jr. and H. F. Kuhfuss, J. Appl. Polym. Sci., Chem. Ed., 25, 1685 (1980).

8. W. J. Jackson Jr., Br. Polym. J., 12, 154 (1980).

9. T. S. Chung, Polym. Eng. Sci., 26, 901 (1986).

10. Y. Kato, S. Endo, K. Kimura, Y. Yamashita, H. Tsugita, and K. Monobe, Kobunshi Ronbunshu, 44, 35 (1987).

11. Y. Yamashita, Y. Kato, K. Kimura, H. Tsugita, and K. Monobe, Kobunshi Ronbunshu, 44, 41 (1987).
12. Y. Kato, Y. Yamashita, K. Kimura, S. Endo, and K. Kajisaki, Kobunshi Ronbunshu, 45, 973 (1988).

13. Y. Yamashita, Y. Kato, S. Endo, and K. Kimura, Makromol. Chem., Rapid Commun., 9, 687 (1988).

14. Y. Kato, Y. Yamashita, K. Kimura, S. Endo, and T. Ohata, Kobunshi Ronbunshu, 47, 583 (1990).

15. K. Kimura, S. Endo, Y. Kato, and Y. Yamashita, Polymer, 34, 1054 (1993).

16. K. Kimura, S. Endo, Y. Kato, and Y. Yamashita, Polymer, 35, 123 (1994).

17. K. Kimura and Y. Yamashita, Polymer, 35, 3311 (1994).

18. K. Kimura, Y. Kato, T. Inaba, and Y. Yamashita, Macromorecules, 28, 255 (1995).

19. Y. Yamashita and K. Kimura, in "Polymeric Materials Encyclopedia", J. C. Salamone, Ed., CRC Press, Boca Raton, FL, 1996, p 8707.

20. H. R. Kricheldorf and A. Conradi, Macromolecules, 22, 14 (1989).

21. G. Schwarz and H. R. Kricheldorf, Macromolecules, 24, 2829 (1991).

22. H. R. Kricheldorf, G. Schwarz, T. Adebahr, and D. J. Wilson, Macromolecules, 26, 6622 (1993).

23. H. R. Kricheldorf, G. Schwarz, and T. Adebahr, Polym. Prepr., (Am. Chem. Soc., Div. Polym. Chem.), 34(1), 768 (1993).

24. G. Schwarz and H. R. Kricheldorf, Macromolecules, 28, 3911 (1995).

25. H. R. Kricheldorf, G. Schwarz, and F. Ruhser, Macromolecules, 24, 3485 (1991).

26. H. R. Kricheldorf, O. Struve, and G. Schwarz, Polymer, 37, 
4311 (1996).

27. A. K. Sarker, K. Kimura, F. Yokoyama, and Y. Yamashita, High Perform. Polym., 13, S351 (2001).

28. K. Kimura, A. K. Sarker, H. Ohmori, and Y. Yamashita, J. Appl. Polym. Sci., (submitted).

29. W. K. Burton, N. Cabrera, and F. C. Frank, Phil. Trans. R.
Soc. Lond. A, 243, 299 (1950).

30. F. C. Frank, Advances in Physics, 1, 91 (1952).

31. R. B. Richards, Trans. Faraday Soc., 42, 10 (1946).

32. H. C. Rain, R. B. Richard, and H. Ryder, Trans. Faraday Soc., 41, 56 (1945). 